\title{
Simulation of Tert-Butyl Alcohol Forming Process by Slurry Catalytic Distillation with Custom Kinetic Program
}

\author{
Zhenya Duan', Shaopu Li', Lei Wang1, Yuan Cheng' ${ }^{2}$, Jingtao Wang', Junmei Zhang2* \\ ${ }^{1}$ College of Electromechanical Engineering, Qingdao University of Science and Technology, Qingdao, China \\ ${ }^{2}$ College of Chemical Engineering, Qingdao University of Science and Technology, Qingdao, China \\ ${ }^{3}$ School of Chemical Engineering and Technology, Tianjin University, Tianjin, China \\ Email: "jmzhang@qust.edu.cn
}

Received 4 February 2016; accepted 16 April 2016; published 19 April 2016

Copyright $@ 2016$ by authors and Scientific Research Publishing Inc.

This work is licensed under the Creative Commons Attribution International License (CC BY).

http://creativecommons.org/licenses/by/4.0/

(c) () Open Access

\section{Abstract}

Producing tert-butyl alcohol (TBA) by slurry catalytic distillation is a green new technology. In order to provide reference data for this production process, this paper applied advanced simulation software Aspen to simulate and optimize the slurry catalytic distillation process of producing TBA. And the kinetics equation of isobutylene hydration which is catalyzed by cation exchange resin in continuous stirred tank reactor (CSTR) is used to display the reaction process. Appropriate theoretical plate number of rectifying section, reaction section and stripping section, reflux ratio and liquid hold-up are obtained by the analog computation. Under this process condition, the conversion rate of isobutylene is $\mathbf{8 2 . 5 3 \%}$; the mole fraction of TBA in the bottom discharging is 82.5\%.

\section{Keywords}

Slurry, Catalytic Distillation, Hydration of Isobutylene, Analog Computation

\section{Introduction}

TBA is an important organic chemical raw material which is used widely in chemical industries. For instance, it is used to add into gasoline to increase the concentration of octane; it is produced to be used as wax solvent, paint solvent, medical solvent and also applied to synthesize synthetic musk [1]. The raw material of producing TBA includes isobutylene and water. And the reaction mainly happens in liquid phase, but low solubility of

${ }^{*}$ Corresponding author.

How to cite this paper: Duan, Z.Y., Li, S.P., Wang, L., Cheng, Y., Wang, J.T. and Zhang, J.M. (2016) Simulation of Tert-Butyl Alcohol Forming Process by Slurry Catalytic Distillation with Custom Kinetic Program. Advances in Chemical Engineering and Science, 6, 140-148. http://dx.doi.org/10.4236/aces.2016.62016 
isobutylene in water may cause low conversion rate of isobutylene. In order to increase the conversion rate of isobutylene and the yield of TBA, slurry catalytic distillation is utilized in the production of TBA [2]. In catalytic distillation, catalytic reaction and distillation are combined together organically. Compared with the traditional reaction and distillation, it has many advantages such as high conversion rate, high selectivity, low energy consumption and low investment. The catalyst used in slurry catalyst distillation is small size particle or ultra-micro particle which can realize the circulation flow with the liquid phase; it has many advantages. For example it can be replaced without parking, it don't need to be separated from solid-liquid system, it has high catalyst efficiency and it is easy to be industrialized. The catalyst is small size particle or ultra-micro particle. As a result, the resistance of mass and heat transfer between liquid phase and catalyst can be reduced greatly, which will promote reaction rate.

In order to conduct the research of process of producing TBA by slurry catalytic distillation, this paper applied advanced simulation software Aspen to simulate and optimize the forming process of TBA by slurry catalytic distillation. To reflect the slurry catalytic distillation process authentically, the kinetics equation of isobutylene hydration reaction catalyzed by fine powder of ion exchange resin is applied by the Fortran interface of Aspen Plus.

\section{Kinetics of Forming TBA by Isobutylene Hydration Reaction}

TBA was traditionally produced by the indirect hydration of isobutylene, catalyst is $50 \%-65 \%$ aqueous solution of sulfuric acid [3]. However, there are many problems such as corrosion of equipment and environment pollution because of the utilization of sulfuric acid. So it is gradually substituted by solid catalyst which catalyzes the forming reaction of TBA by hydration of isobutylene, among the solid catalysts, Amberlyst 15 and Dowex 50 ion-exchange resin have many advantages such as high activity, high selectivity, long life expectancy, no corrosion, high mechanical strength and strong temperature resistance, so they are applied in the highest frequency. In literature [4], NKC-9 fine particle is used as catalyst, and the reaction kinetics equation of isobutylene hydration is investigated comprehensively in CSTR. NKC-9 macro-reticular cation exchange resin is manufactured by Nankai University whose characters and properties are similar to Amberlyst 15.

Reaction kinetics equation of isobutylene hydration in literature [4]:

$$
r=\frac{c \beta k\left(C_{B} C_{W}-C_{A} / K_{C}\right)}{\left(1+K_{W} C_{W}\right)^{2}}
$$

Relationship between the inhibition coefficient of TBA and temperature in the formula above:

$$
K_{w}=\mathrm{e}^{7.0143-\frac{5504.15}{T}}
$$

Relationship between equilibrium constant and temperature:

$$
K_{c}=\mathrm{e}^{\frac{6311.42}{T}-22.731}
$$

Relationship between reaction rate constant and temperature:

$$
k=k_{0} \mathrm{e}^{-\frac{E_{a}}{R T}}
$$

Activation energy in the formula:

$$
E a=122356.182 \mathrm{~J} / \mathrm{mol}
$$

Relationship between pre-exponential factor and the concentration of catalyst:

$$
k_{0}=4.3409 \times 10^{12}+4.1422 \times 10^{11} C_{\text {cat }}
$$

$\beta$ is the influence coefficient of TBA, because the forming reaction of TBA by hydration of isobutylene is reversible, so the existence of TBA can increase the reverse reaction rate [5], and its acceleration effect is linear, but the solubility of isobutylene will increase exponentially due to the existence of TBA in the liquid phase [6] [7], and also the positive reaction rate will increase exponentially. When a little TBA is added before reaction to increase the solubility of isobutylene in the liquid phase, $\beta=1$ [8] [9]. 


\section{Simulation for the Slurry Catalytic Distillation Process}

The flow process of forming TBA by slurry catalytic distillation is shown in Figure 1.

Two feed streams of the slurry catalytic distillation column are isobutylene and water. The purity of isobutylene is $99.99 \%$, and there is catalyst particle in the water. The positions of two discharging streams are the top of column (main component is isobutylene) and the bottom of column (main components are TBA and a little water).

Hypotheses in the simulation process:

(1) Without regarding to the chemical reaction in the gas phase and the side reaction in hydration of isobutylene;

The middle part of reactive distillation column is the reaction section. Catalyst particle mainly exists in liquid phase, and the hydration of isobutylene is hard to proceed in gas phase. The side effects in the hydration of isobutylene are the reaction of di-tert-butyl ether catalyzed by acid and the dimer reaction of isobutylene, the degree of this kind of side effect are all very small, so the side reactions and by-products are not defined in the analog computation.

(2) Without regarding the mass transfer resistance in the internal of catalyst fines, and the slurry is considered as homogeneous.

Although the solubility of isobutylene is low in water, it can be increased greatly by a little TBA in the raw material. At the same time, with the strong stirring action of rising vapor in the liquid on plates, isobutylene can fully mix with water. In addition, the average grain diameter of cation exchange resin catalyst fines is $10 \mu \mathrm{m}$, it has been proved by experiment [4] that the utilization of catalyst in such diameter can eliminate the effect of internal diffusion. The catalyst fines distribute evenly in the liquid phase, then form slurry with the liquid phase. At the same time form a circular flow with the liquid phase in the column, so the effect of external diffusion is left out, and the reaction slurry can be regarded as homogeneous.

(3) The liquid hold-up on each plate in the reaction section is the same.

Due to the condition on each plate in the reaction section is identical, the amount of liquid hold-up on each plate in the reaction section is supposed to be the same.

\subsection{Reaction Kinetics in the Simulation Process}

In the Radfrac module of Aspen Plus, there are three kinds of models: conversion rate model, equilibration model and kinetics model [10]. The relationship between conversion rate and temperature or conversion rate of each plate is need to be given in the conversion rate model. The equilibration reaction model is used in system where the reaction can reach equilibration in a very short time. In the process of forming TBA by slurry catalytic distillation, isobutylene hydration exists on many plates in the reaction section of distillation column whose reaction rate is low (in the reaction kettle, if the content of acidic ion-exchange resin is $2 \mathrm{wt} \%$, the equilibrium conversion rate in one hour is $30 \%$ at $80^{\circ} \mathrm{C}$ [4]), which has a strong relationship with temperature and component content. So it is not suitable to use the conversion rate model and equilibration reaction model. As a result, the kinetics model is applied in this paper. The internal definition of the reaction kinetic equation for ASPEN:

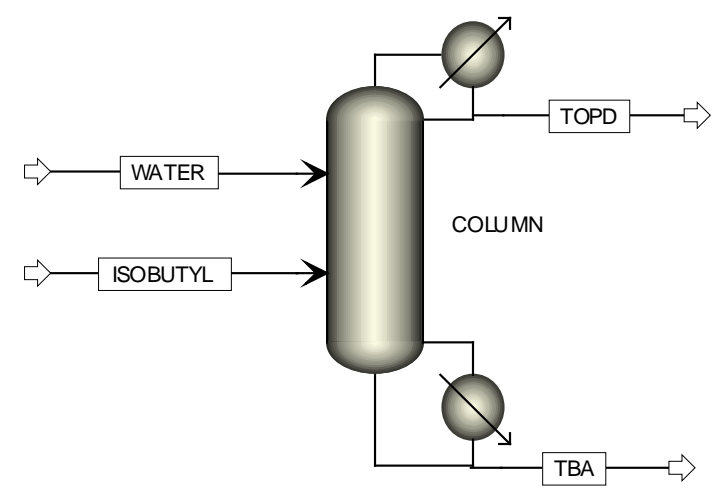

Figure 1. The flow process of forming TBA by slurry catalytic distillation. 


$$
r=k\left(T / T_{0}\right)^{n} \mathrm{e}^{-(E / R)\left[1 / T-1 / T_{0}\right]} \prod_{i=1}^{N} C_{i}^{\alpha_{i}}
$$

There are some differences between this formula and the kinetic equation for hydration of isobutylene, so the kinetic equation of isobutylene hydration is applied in this paper to simulate the reaction process of slurry catalytic distillation, the invoking process of the custom reaction equation in Aspen is as follows. At first, copy the compiled Fortran file "usrknt.f" of the reaction kinetics program into the working directory of Aspen, then open up the Aspen Plus Simulation Engine through the start menu, enter command aspcomp usrknt.f and asplink usrknt.obj usrknt.dll respectively, after compiling and linking, its corresponding dynamic link library can be successfully created, then copy the object file it produced “*.obj” or the dynamic linking library “*.dll” into the perform directory of Aspen Plus, as a consequence, the user's kinetic program can be invoked in the RadFrac module of Aspen Plus. In the RadFrac module, "Use user kinetic subroutine” is selected as the reaction kinetics type, the name of user's kinetic program is also needed to be typed into the input box in the Subroutine tab.

\subsection{Thermodynamic Model in Simulation Process}

The main components in the catalytic distillation column are $\mathrm{H}_{2} \mathrm{C}=\mathrm{C}\left(\mathrm{CH}_{3}\right)_{2},\left(\mathrm{CH}_{3}\right)_{3} \mathrm{COH}$ and $\mathrm{H}_{2} \mathrm{O}$, which is a high non-ideal system. NRTL, UNIQUAC, WILSON constitutive equation can be utilized to calculate the liquid property of the system. The initial analog result indicates that there is no distinct difference between the results of these three equations, but it is easier to converge using the UNIQUAC equation. So UNIQUAC equation is used in the analog computation. Relative parameters and the property of pure components can be found in the database of Aspen.

\section{Simulation Result and Discussion}

The boiling point of isobutylene is $-6.9^{\circ} \mathrm{C}$ under the atmosphere. Under the pressure of 3 atm, the boiling point of isobutylene is close to room temperature. Taking the requirement of operating pressure for equipment into account, the overhead pressure and feed-in pressure are all set as $3 \mathrm{~atm}$, is fed at the bubble point. 1:1 is chosen as the feed flow rate ratio of water and isobutylene in this paper. Referring to the experimental data in literature [4], simulation and optimization of the effect of parameters (theoretical plate number, operating pressure, liquid hold-up. etc.) on the reaction in the process of forming TBA by slurry catalytic distillation are completed. Conclusions can be reached as follows.

\subsection{Theoretical Plate Number}

In order to investigate the effect of theoretical plate number in rectifying section, reaction section and stripping section on the purity of TBA and conversion rate of isobutylene at the bottom of column. When the theoretical plate number in a certain section is changed, fixing the operating condition and theoretical plate number in other sections, the result which mirrors effect of theoretical plate number on separation is shown in Figures 2-4.

The process condition of catalytic distillation is as follows. Mole flow of isobutylene is $50 \mathrm{kmol} / \mathrm{h}$, mole flow of water is $50 \mathrm{kmol} / \mathrm{h}$, feed-in temperature is $298.15 \mathrm{~K}$, column pressure is $303.975 \mathrm{KPa}$, the theoretical plate number in reaction section and stripping section are separately fixed as 13 and 5 respectively.

Figure 2 shows the effect of theoretical plate number of rectifying section on concentration of TBA and conversion rate of isobutylene at the bottom of column. As it is shown in Figure 2, when the theoretical plate number of rectifying section exceeds 3, the purity of TBA and the conversion rate of isobutylene at the bottom of column will be remained on a high level. Taking stability into account, the theoretical plate number in rectifying section is set as 4 . When the theoretical plate number of rectifying section and stripping section are separately set as 4 and 5, the effect of theoretical pate number in reaction section on reaction is shown in Figure 3. Due to the low solubility of isobutylene in water, the reaction rate is relatively low. In order to increase the conversion rate of reaction, the theoretical pate number of reaction section is set as 12 to 17 . As it is shown in Figure 3 , with the increasing of theoretical plate number in reaction section, some TBA and water will be evaporated from the top of the column which is adverse to separation. As a result, the theoretical plate number in reaction section is set as 15 . When there are 4 plates in rectifying section and 5 plates in reaction section, the effect of theoretical plate number in rectifying section is shown in Figure 4. The water-TBA azeotrope system whose relative vola- 


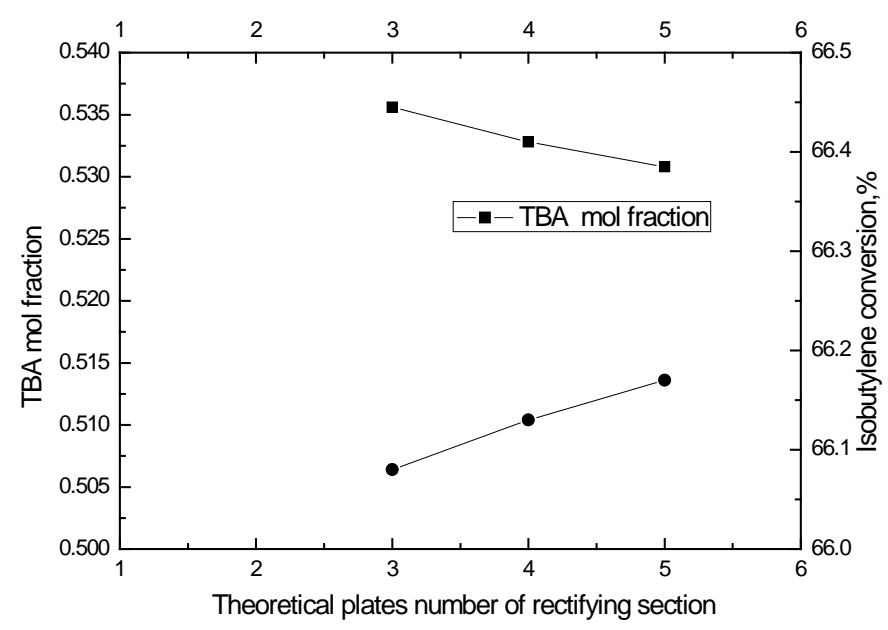

Figure 2. The effect of theoretical plate number in rectify section on products' quality.

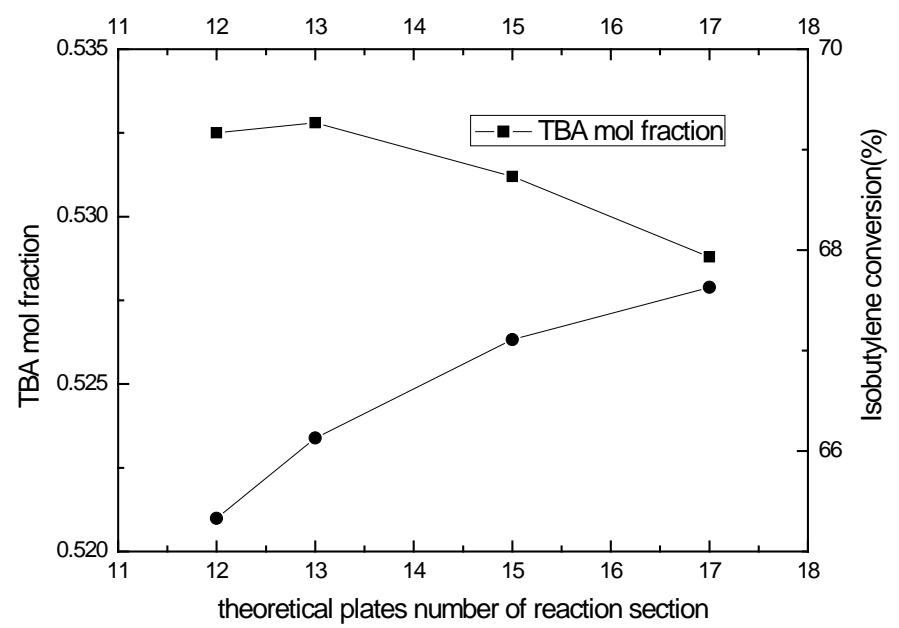

Figure 3. The effect of theoretical plate number in reaction section on products' quality.

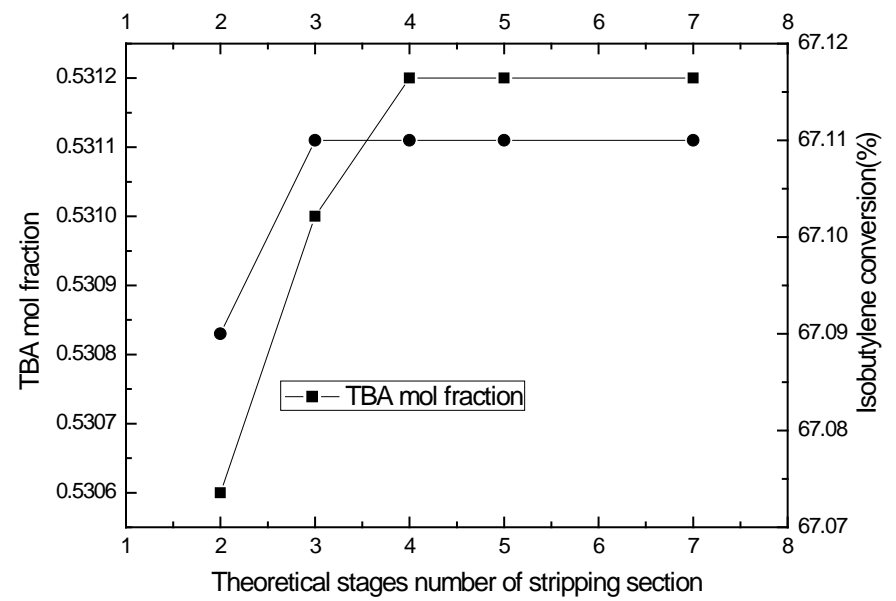

Figure 4. The effect of theoretical plate number in stripping section on products' quality. 
tility is higher is easy to be separated, so only few theoretical plates are needed. As it is shown in Figure 4, promotion of theoretical plate number in stripping section will not make much difference to separation. But considering the effect of theoretical plate number in stripping section under different reflux ratio on reaction conversion rate and separation, the theoretical plate number in stripping section is set as 4 .

\subsection{Reflux Ratio}

Reflux ratio is an important parameter in distillation unit operation. In this paper, other operating parameters are fixed to investigate the effect of reflux ratio on mole content of TBA at the bottom discharging of catalytic distillation column. The analysis result is shown in Figure 5.

The simulation result indicates that: the mole content of TBA at the bottom discharging increases with the increasing of reflux ratio. When the reflux ratio arrives at 15 , the increasing will be slower. But when the reflux ratio approach to 19 , content of TBA at the bottom discharging will decrease suddenly. Taking the stability of practical operation into consideration, reflux ratio is set as 15 in this paper.

\subsection{Liquid Hold-Up of Reaction Section}

In reaction section, catalytic reaction time is determined by the contact time of gas and liquid. Liquid hold-up is an important parameter that influences the contact time of gas and liquid. In this paper, the effect of liquid each plate hold-up of reaction section on conversion rate and separation of isobutylene is investigated. The process condition of catalytic distillation is as follows: mole flow of isobutylene is $50 \mathrm{kmol} / \mathrm{h}$, mole flow of water is 50 $\mathrm{kmol} / \mathrm{h}$, feed-in temperature is $298.15 \mathrm{~K}$, column pressure is $303.975 \mathrm{KPa}$, theoretical plate number of rectifying section, reaction section, stripping section are 4, 15 and 4, reflux ratio 15. Detailed data is shown in Figure 6 and Figure 7. As it is shown in Figure 6, the conversion rate of isobutylene increases with the increasing of liquid hold-up on a single plate. When liquid hold-up on a single plate in reaction section exceeds $40 \mathrm{~L}$, the effect of liquid hold-up on conversion rate of isobutylene turned out to be small. But at the same time, liquid load in reaction section increases with the increasing of liquid hold-up. So liquid hold-up should be no more than $40 \mathrm{~L}$. As it is shown in the data in Figure 6, when liquid hold-up exceeds $30 \mathrm{~L}$, purity of isobutylene at the top of column may be significantly reduced with the increasing of liquid hold-up (adverse to the recycle of isobutylene) and some TBA may be mixed into product at the top of column. And the mole fraction of TBA at the bottom distillate won’t increases significantly. From what mentioned above, liquid hold-up on a single plate in reaction section is set as $25 \mathrm{~L}$ in this paper.

\subsection{Position and Control of Sensitive Plate}

Determining the location of sensitive plate is an important part in maintaining normal operation of distillation column. When external factors changes, temperature and composition of sensitive plate may change significantly. In this paper, the temperature distribution of the whole tower is simulated under reflux ratio 12,15 , 18. It is

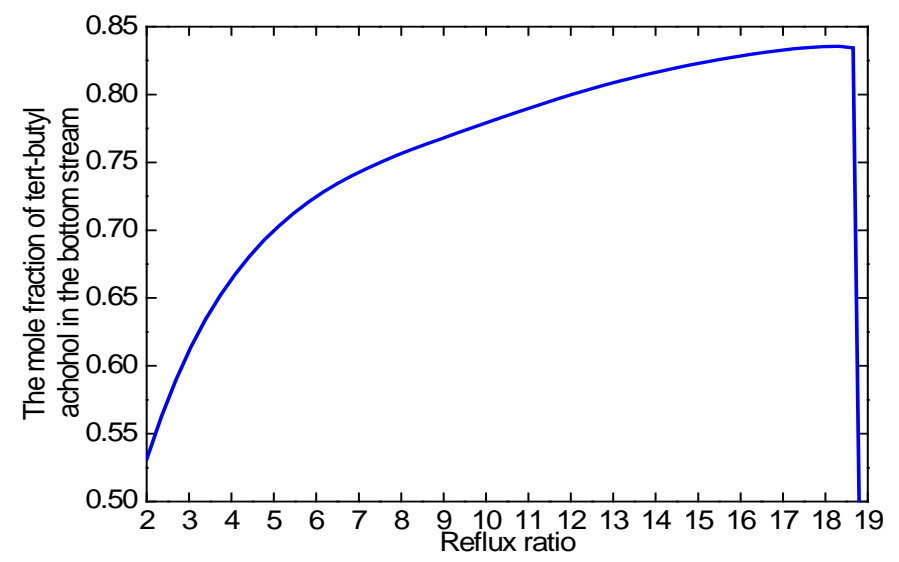

Figure 5. The effect of reflux ratio on the mole fraction of tert-butyl alcohol in the bottom stream. 


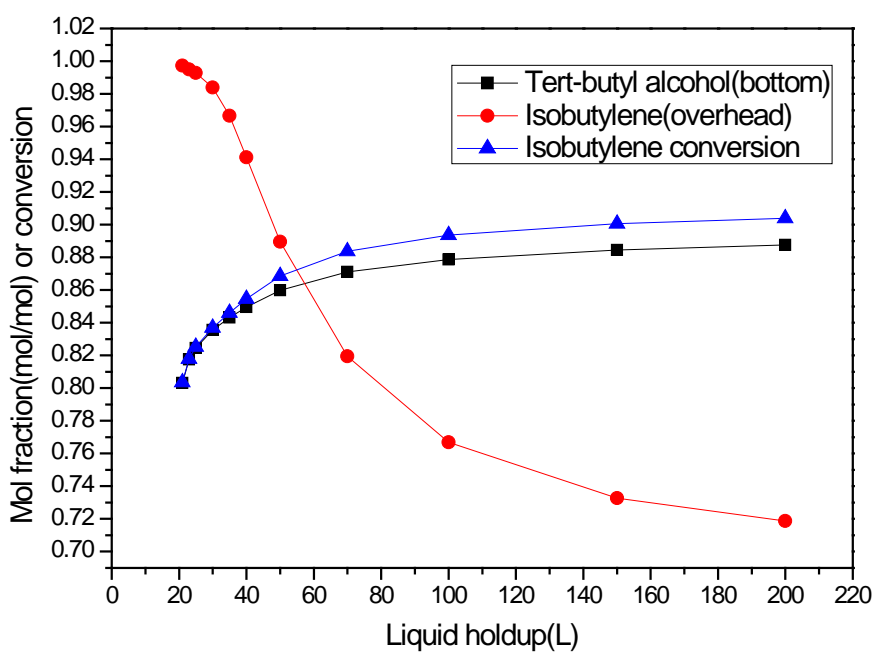

Figure 6. The impact of liquid holdups on separation and conversion rate of isobutylene.

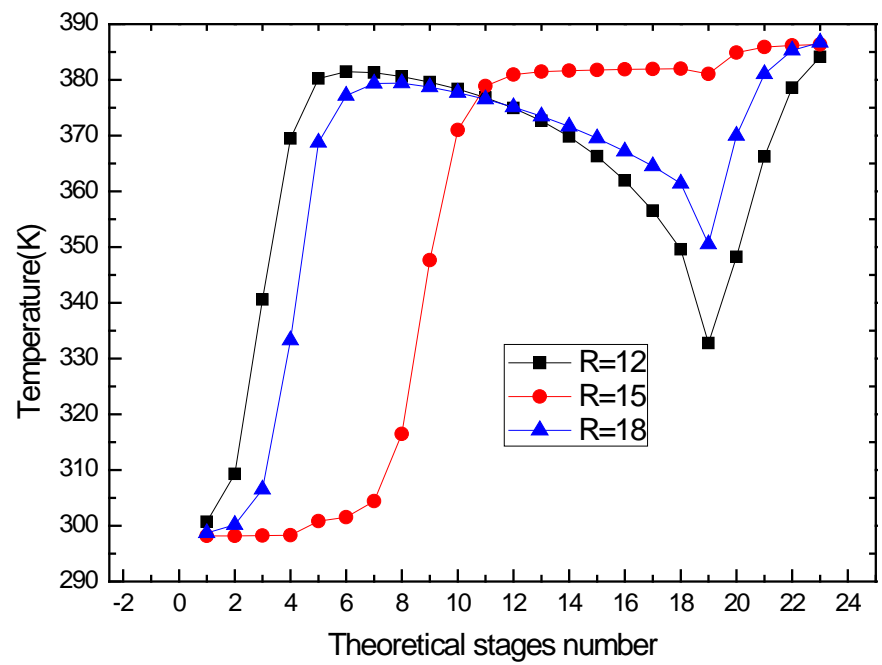

Figure 7. The effect of reflux ratio on the temperature distribution along the catalysis rectification column.

shown in the temperature distribution Figure 7, under different reflux ratio, there are two parts in catalytic distillation column, in which temperature changes greatly. One is from plate NO.3 to NO.10 (the upper part of reaction section), the other is from plate NO.17 to NO.21 (the lower part of reaction section). These two parts are the most sensitive parts to the changing of external interference. As a result, both the top and the bottom of column should be controlled through two temperature control circuits. The first control circuit is to maintain the temperature at some points in the mid-upper part of reaction section constant by means of controlling the flow rate of top column. The second control circuit is to maintain the temperature at some points in the lower part of reaction section constant by adjusting the feed-in flow rate of the isobutylene.

As it is shown in analog result which indicates the effect of parameters (plate number, reflux ratio, liquid hold-up, etc.) on reaction and separation, the appropriate process conditions are as follows. Molar flow of isobutylene is $50 \mathrm{kmol} / \mathrm{h}$, molar flow of water is $50 \mathrm{kmol} / \mathrm{h}$, feed-in temperature is $298.15 \mathrm{~K}$, column pressure is 303.975 KPa, theoretical plate number of rectifying section, reaction section, stripping section are 4, 15 and 4, feed-in position of water is plate NO.5, feed-in position of isobutylene is plate NO.19, reflux ratio is 15 and liquid hold-up is $25 \mathrm{~L}$. Under such process condition, conversion rate of isobutylene is $82.53 \%$, mole fraction of TBA in the bottom discharging is $0.5 \%$, mole fraction of water is $17.4 \%$. Molar fraction of each component 


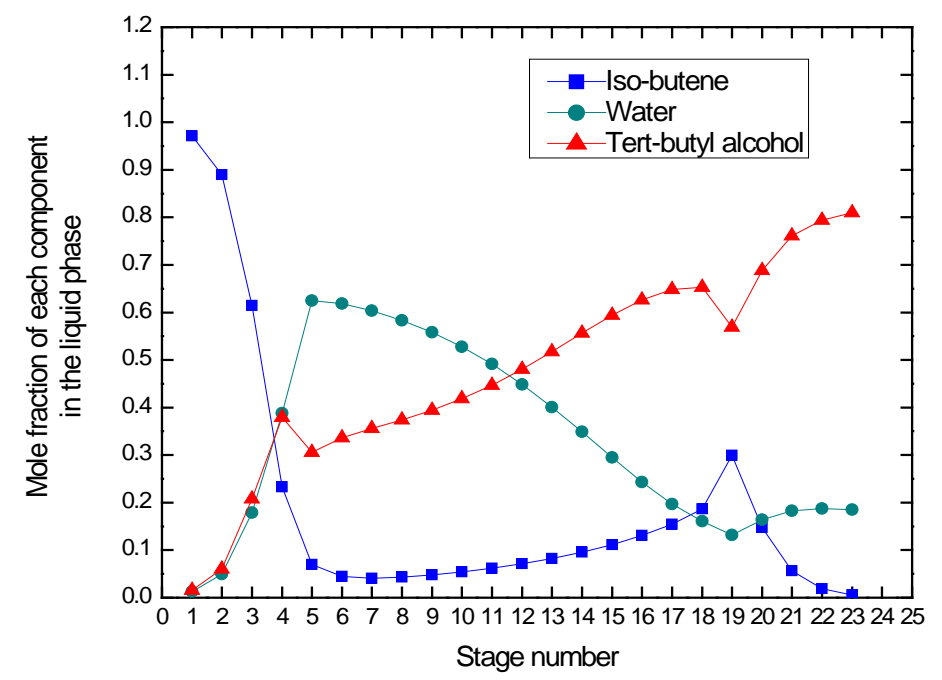

Figure 8. The mole fraction of each component in liquid phase along the column.

along the column can be seen in Figure 8.

As it is shown in the figure, in liquid phase of distillation column, concentration of isobutylene is high in the top of column, due to its high relative volatility. In the liquid phase of rectifying section and stripping section of catalytic distillation column, changing trend of mole fraction of water and TBA are almost consistent. While in liquid phase of reaction section, changing trend of water and TBA is totally different. It is mainly because TBA and water can form an azeotrope. But in the reaction section, there will be a drop in the molar concentration of water and an increase in the molar concentration of TBA in reaction section, which destroy the azeotropy of TBA and water, so it presents a trend in Figure 8.

\section{Conclusions}

In this paper, through referring the kinetic equation of isobutylene hydration under the effect of fine particle in other literature, we get the kinetic equation and write it into the Aspen users' kinetic interface program with Fortran language. The invoking of Aspen user's kinetic program is realized successfully by compiling and linking. An optimization scheme is obtained by analog computation and optimizing of the slurry catalytic distillation process in which TBA is produced.

(1) Theoretical plate number in each section of reactive distillation column are determined: 4 plates in rectifying section, 15 plates in reaction section, 4 plates in stripping section, 23 theoretical plates in total.

(2) After fixing plate number, the optimal reflux ratio can be obtained through sensitivity analysis of Aspen which is 15.

(3) To increase the boiling point of isobutylene, pressurized distillation is needed and top pressure is set as 3 atm.

(4) Water flows into the tower at the upper part of reaction section whose temperature is $25^{\circ} \mathrm{C}$. Isobutylene flows into the tower at the lower part of reaction, under the pressure of $3 \mathrm{~atm}$, at the bubble point.

(5) Through simulation analysis, it can be determined that both the upper and lower part of reaction section are sensitive to temperature changing. In practical production, real-time monitoring and control should be performed on these two parts.

\section{Acknowledgments}

This work reported here was supported by the Science and Technology Planning Project of Shandong Provincial Education Department (No.J12LD15 and J15LC16), State Environmental Protection Key Laboratory of Sources and Control of Air Pollution Complex (No. SCAPC201405), and the Shandong Province Taishan Scholar engineering under special funding. We express our grateful thanks to them for their financial support. 


\section{References}

[1] Elavarasan, P., Kondamudi, K. and Upadhyayula, S. (2011) Kinetics of Phenol Alkylation with Tert-Butyl Alcohol Using Sulfonic Acid Functional Ionic Liquid Catalysts. Chemical Engineering Journal, 1, 340-347.

[2] Doraiswamy, L.K. and Sharma, M.M. (1984) Heterogeneous Reaction. Wiley, New York.

[3] Gehlawat, J.K. and Sharma, M.M. (1968) Absorption of Isobutylene in Aqueous Solutions of Sulphuric Acid. Chemical Engineering Science, 23, 1173-1180. http://dx.doi.org/10.1016/0009-2509(68)89024-4

[4] Wang, Y. (2002) The Study of Kinetics of Isobutylene Hydration Reaction Catalyzed by Fine Power of Ion Exchange resin. Master. Dissertation, Tianjin University, Tianjin.

[5] Dellon, A., Torck, B. and Hellin, M. (1986) Equilibrium Constant for the Liquid-Phase Hydration of Isobutylene over Ion-Exchange Resin. Industrial and Engineering Chemistry Process Design and Development, 25, 889. http://dx.doi.org/10.1021/i200035a009

[6] Leung, P.C., Zorrilla, C., Puigjaner, L., et al. (1987) Solubilities and Enthalpies of Absorption of Isobutene into Tert-Butyl Alcohol-Water Mixtures. Journal of Chemical and Engineering Data, 32, 169. http://dx.doi.org/10.1021/je00048a012

[7] Velo, E., Puigjaner, L. and Recasens, F. (1988) Inhibition by Product in the Liquid-Phase Hydration of Isobutene to Tert-Butyl Alcohol: Kinetics and Equilibrium Studies. Industrial and Engineering Chemistry Process Design and Development, 27, 2224. http://dx.doi.org/10.1021/ie00084a006

[8] Caceres, E., Puigjaner, L. and Recasens, F. (1988) A Trickle-Bed Process for Hydration of Isobutene to Tert-Butyl Alcohol: A Study of Reactor Performance. The Chemical Engineering Journal, 37, 43.

[9] Leung, P., Zorrilla, C., Recasens, F., et al. (1986) Hydration of Isobutene in Liquid-Full and Trickle-Bed Reactors. AIChE Journal, 32, 1893. http://dx.doi.org/10.1002/aic.690321109

[10] Luyben, W.L. (2006) Distillation Design and Control Uesing ASPENTM Simulation. John Wiley \& Sons, Inc., Hoboken. 